\title{
In Situ Material Removal over Many Orders of Magnitude
}

\author{
M. Straw, D. Phifer \\ FEI Company, 5350 NE Dawson Creek Dr., Hillsboro, OR 97124
}

The industrial success of focused ion beam technology is, in large part, attributable to the development by IC manufacturers of applications that rely on the ability to remove material - applications that enable defect analysis, failure analysis, circuit edit, statistical process control, and metrology. In the past, these applications required only small volumes of material to be removed with relatively low material removal rates acceptable. However, the growing need to combine functionally diverse, often non-digital technologies and traditional CMOS-based digital processing and storage via 3D IC integration requires applications that call for larger volumes and higher rates of material removal [1]. As a result, providing users the ability to manipulate matter over a larger range of length scales in a single tool is becoming more and more recognized as a value added proposition that can make critical processes faster, cheaper, and more reliable.

To this end, we have developed a triple beam tool that combines a FEG SEM, a Ga LMIS FIB, and a Ti:Sapphire ultrashort pulsed laser (UPL) capable of delivering a $150 \mathrm{fs}, 1 \mathrm{~mJ}$ pulse with a center wavelength of $775 \mathrm{~nm}$ at a repetition rate of $1 \mathrm{kHz}$. The UPL is delivered via a laser injection port (LIP) consisting of a window through which the beam enters, $\mathrm{X}$ and $\mathrm{Y}$ control for beam positioning, focus control, a bellows, a beam tube through which the unfocused beam propagates, and a microscope objective to focus the beam on the sample. The laser is located on an adjacent optical table and the beam is steered through the window by a series of mirrors, one of which is attached to the LIP. Dynamic power modulation is achieved by a typical analyzer-polarizer combination located on the optical table. The laser power can be further modulated via a series of ND filters and/or through the front panel control of the laser itself. The laser power is monitored via a silicon detector located just prior to the input window. The interior of the tool's chamber is shown is shown in Figure 1. Via our X, Y, and Z control, the UPL beam can be aligned to be coincident with the FIB and SEM beams.

A natural synergy between these three technologies arises from their coincident delivery. UPLs are well known for their ability to rapidly remove large volumes of nearly any material with little or no concomitant damage to nearby structures. Indeed, we have demonstrated in situ material removal rates in excess of $7.8 \times 10^{6} \mu \mathrm{m} /$ second (see Fig. 2). Once exposed by the laser, features of interest can be crosssectioned and polished atomically smooth via FIB. Finally, SEM can be employed to collect high resolution imaging data. The demonstrable ability to perform complex, multi-stage processes on a single tool will inevitably yield improvements in the speed, cost, and repeatability of said process.

While our primary motivation for exploring UPL technology is to enable applications that require the rapid removal of large volumes of material, a host of unique microanalysis and material characterization techniques become available via the use of UPLs such as laser induced breakdown spectroscopy (LIBS) and laser induced X-ray spectroscopy. Beyond analysis and characterization, additive techniques such as laser-induced plasma-enhanced deposition and pulsed laser deposition are also currently being explored and promise to enable relatively pure, large area deposits of metals and dielectrics.

Due to the wide range of techniques available via ultrashort pulsed lasers, their integration with our Small DualBeams (SDB) promises to provide additional manufacturing, prototyping, analysis, and characterization capabilities. Furthermore, applications that are impossible or impractical using FIB become feasible upon the introduction of UPLs. Typically, this applies to applications that require very high rates of material removal or relatively large area depositions. However, it is not unlikely that new applications demonstrating a "whole-greater-than-the-sum-of-its parts" capability will be uncovered as $\mathrm{UPL}+\mathrm{SDB}$ matures. 


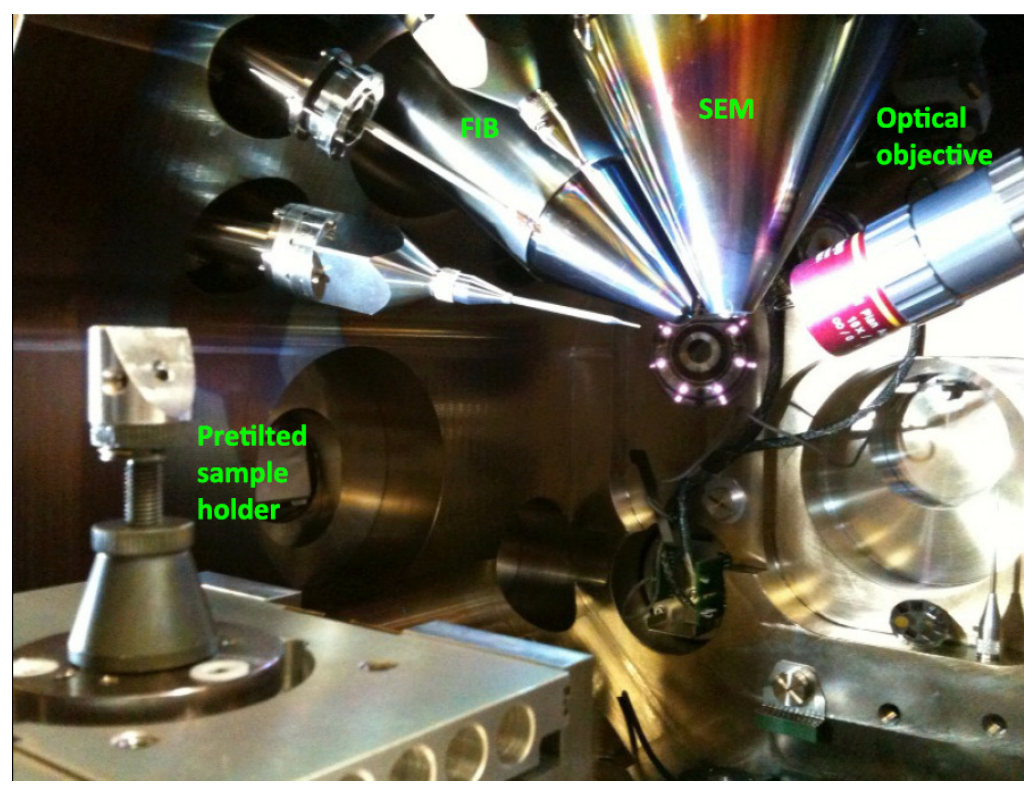

Figure 1. Small DualBeam chamber interior with integrated UPL.

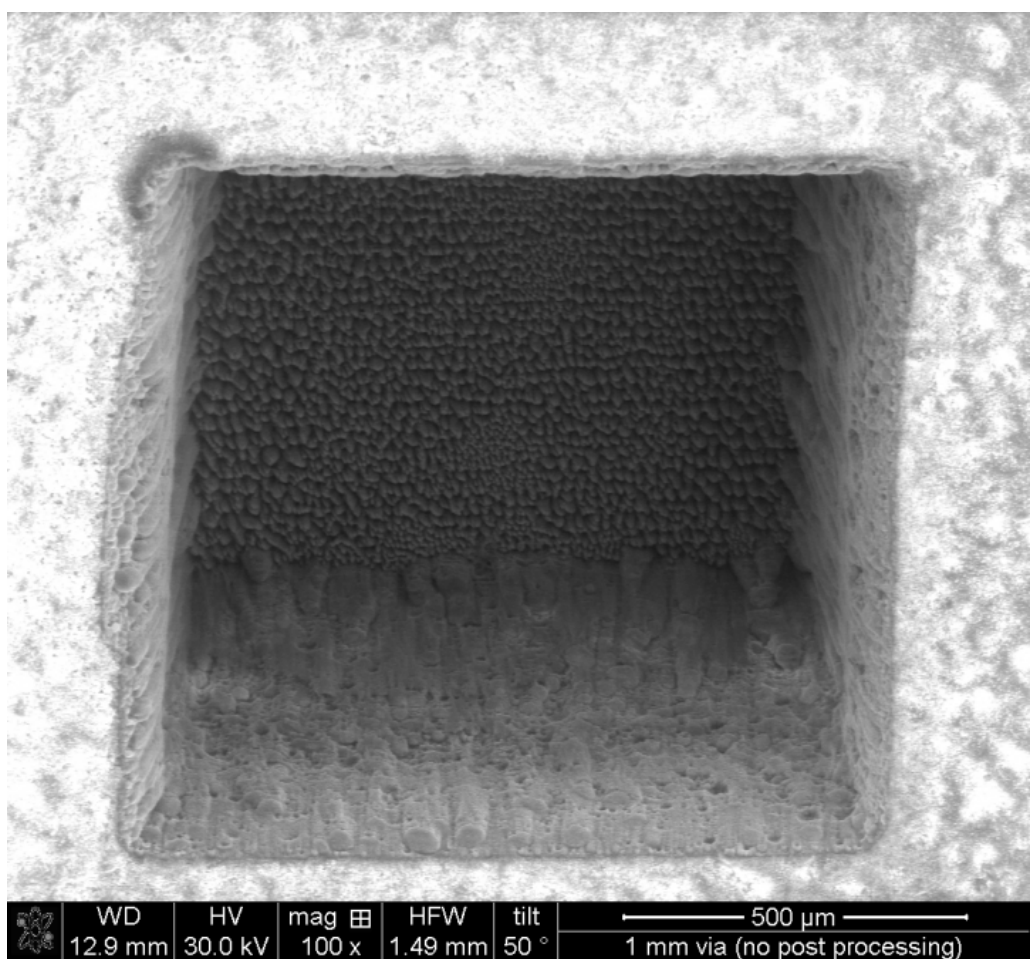

Figure 2. Silicon die accessed through the epoxy and glass ball package. $0.75 \mathrm{~mm}^{3}$ of material removed in $96 \mathrm{sec}$ (material removal rate $>7.8 \times 10^{6} \mu \mathrm{m}^{3} / \mathrm{sec}$ ). No ex situ post-processing performed. Processing was stopped on top surface of Si die.

1. Arden, W., et al., More-than-Moore. International Roadmap Comittee (IRC) of the International Roadmap for Semiconductors (ITRS), www.itrs.net, 2010. 